\title{
Effect of hard segment content and carbon-based nanostructures on the kinetics of flexible polyurethane nanocomposite foams
}

M. Mar Bernal, ${ }^{* a}$ Mario Martin-Gallego, ${ }^{a}$ Laura J. Romasanta, ${ }^{a}$ Anne-Cecile Mortamet, ${ }^{b}$ Miguel A. López-Manchado, ${ }^{a}$ Anthony J. Ryan, ${ }^{b}$ and Raquel Verdejo $* a$

anstituto de Ciencia y Tecnología de Polímeros, ICTP-CSIC

Consejo Superior de Investigaciones Científicas, Juan de la Cierva 3, E-28006, Madrid, Spain

${ }^{b}$ Department of Chemistry, University of Sheffield, Sheffield, S3 7HF, United Kingdom

*Corresponding author: Tel: +34 9125875 61. Fax: +34 9156448 53. E-mail address: mbernal@ictp.csic.es $o r \underline{\text { rverdejo@ictp.csic.es }}$ 


\begin{abstract}
Reactive flexible polyurethane (PU) foams were synthesized with two contents of hard segments (HS) and filled with multi-walled carbon nanotubes (MWCNTs), functionalized MWCNTs (f-MWCNTs) and functionalized graphene sheets (FGS). The effect of the HS content and the carbon nanofillers on the kinetics of polymerization and the kinetics of phase-separation have been studied by Fourier transform infrared spectroscopy (FT-IR) and synchrotron small-angle X-ray scattering (SAXS). A slow down on the rate of polymerization and on the development of the polymer structure due to the increase of the HS content and the inclusion of the nanoparticles was observed. Therefore, this work demonstrates that there is a relationship between the kinetics of polymerization and the kinetics of phase separation in flexible PU nanocomposite foams. SAXS data was used to generate 3D microstructures of PU nanocomposite foams and the phase-separated morphology was observed by atomic force microscopy (AFM).
\end{abstract}




\section{Introduction}

Polyurethane (PU) foams are one of the most versatile polymeric materials because they can be formulated to meet specific requirements and, hence, are used in applications as diverse as shock absorbers in vehicles, packages or footwear, thermal insulators in appliances and constructions; and furniture among others.[1, 2] Water-blown PU foams are formed by the simultaneous polymerization and expansion of relatively low molecular weight reactive liquids, resulting in solid cellular materials. Thus, the process has to balance chemical and physical events that can be monitored by the reaction kinetics and the changes in the morphology. $[3,4]$ PU foams are essentially produced by two exothermic reactions between the isocyanate with the hydrogen-active groups of polyol and water. The reaction between the isocyanate and the polyol is called the gelling reaction and forms urethane linkages leading to an increase in the molecular weight. The blowing reaction is produced between the isocyanate and water and forms ureas, also referred as hard segments (HS), and carbon dioxide gas due to the decomposition of an unstable carbamic acid.[5] A segmented block copoly(urethaneurea) is then the result of these two reactions, where the urea groups are covalently bonded to the polyol chains through urethane linkages. Hence, PU foams present a hierarchical structure: the matrix phase-separated block copolymer morphology at the nanoscale and the cellular structure at the microscale. As in the case of all segmented block copolymers, the phase-separated morphology of PU foams has a profound effect on the final properties of the system.

Early studies on the effects of HS on segmented polyurethanes can be found in the literature.[6-9] Synchrotron small-angle X-ray scattering (SAXS) and differential scanning calorimetry (DSC) were used to determine the mechanisms of phase separation during the annealing or cooling from the melt state of segmented polyurethanes.[6-9] These works reported that an increase on the hard segment content reduced the mobility of the system establishing that the kinetic factor, i.e. the viscositymobility-interaction argument, rather than the thermodynamic factor explained the phase separated structure of PU. Hence, the works concluded that this phase-separated morphology is ensured by the thermodynamic incompatibility of the hard and soft segments but it is then controlled by the system viscosity, the hard segments mobility, and the interaction between the hard and the soft segments. A recent work combined synchrotron SAXS and temperature-controlled Fourier Transform infrared (FT-IR) to 
monitor the degree of hard/soft segment segregation as a function of temperature and observed a mixing of the phase-separated segments with increasing temperature.[10] On the other hand, in-situ studies of the structure development of flexible PU using FT-IR and synchrotron SAXS were reported by Ryan and co-workers.[11-13] The combination of both techniques enables the real-time monitoring of the morphological changes of PU foams during its polymerization. These studies confirmed that the thermodynamics of phase separation dominates the kinetics of hydrogen bonding.

Recently, the effect of nanofillers on the foaming evolution of polyurethane foams has been studied.[14-16] These works analyzed the kinetics of the foaming reaction by a qualitative method based on a cup test.[14, 15] Meanwhile, Wilkinson et al.[16] measured the reaction rates of flexible PU foam-layered silicate nanocomposites by quantitative methods based on adiabatic temperature rise and FT-IR spectroscopy. Aside from the method employed to follow the foaming kinetics, these studies observed an acceleration of the reaction rate due to the inclusion of nanoclays and carbon nanofibers.[14-16] In contrast, we qualitatively observed the opposite trend, i.e. a delay on the rate of foaming evolution [17]. Further detailed examination by FT-IR and rheological measurement confirmed the observed delay as a result of kinetic effects imposed in chain mobility by the presence of the CNTs.[18, 19] These studies showed the presence of homogeneously dispersed carbon nanoparticles within the cellular structure of the foams throught a careful analysis of the rheological properties.[18, 19] In the present work, we study the effect of the carbon nanostructures in the matrix phase-separated block copolymer morphology, since it has a profound effect on the final properties of the foam. Hence, we select a fixed loading fraction and two different contents of hard segments and analyze both the kinetics of polymerization and the kinetics of matrix morphology development. The reaction kinetics of PU nanocomposites foams is monitored by FT-IR spectroscopy while the matrix morphology development is investigated by synchrotron SAXS. Visual 3D representations of the PU morphologies are generated from the SAXS data. Finally, the effect of the carbon nanofillers on the phase-separated morphology is analyzed by AFM. 


\section{Experimental}

\subsection{Materials}

The isocyanate used in this study was a methylene diphenyl diisocyanate (MDI) (Voranate M2940) with NCO content of 31.4 wt.-\%, an average functionality of 2.3 and containing high quantities of monomeric diphenylmethane-4,4'-di-isocyanate (51.0 $61.0 \%$ ) while the polyol was a polyether based triol (Voranol 6150) with $\mathrm{OH}$ value of $27 \mathrm{mg} \mathrm{KOH} / \mathrm{g}$ and $\mathrm{MW}$ of $6000 \mathrm{~g} / \mathrm{mol}$, being both products kindly supplied by Dow Plastics. The polyol based triol Voranol CP1421 (OH value: $31 \mathrm{mg} \mathrm{KOH} / \mathrm{g}$ ) was used as a cell-opener in the reaction. The additives used in the formulation were: FASCAT 4202 (dibutyl tin dilaurate from Arkema Inc.) as tin-based catalyst for the gelling reaction, TEDA L-33B (a solution of 33\% triethylendiamine in 1,4-butanediol from Torch Europe B.V.) and NIAX E-A-1 (a solution of 70\% bis(2-dimethylaminoethyl) ether in $30 \mathrm{wt} .-\%$ dipropylene glycol from Momentive Performance Materials Gmbh) as amine derivative catalysts in the water/isocyanate and polyol/isocyanate reactions, balancing the tin catalyst, DEOA (85\% diethanolamine in water) as a cross-linker agent, SH-209 (Momentive Performance Materials Gmbh) as silicone surfactant and distilled water as blowing agent.

Aligned MWCNTs were grown by the chemical vapour deposition (CVD) injection method.[20, 21] A 3 wt.-\% ferrocene/toluene solution was injected into a hot quartz tube reactor $\left(760{ }^{\circ} \mathrm{C}\right)$ at $5 \mathrm{ml} \mathrm{h}^{-1}$ under inert atmosphere. These nanotubes were chemically-treated with a 3:1 concentrated sulphuric-nitric acid mixture and refluxed at $120^{\circ} \mathrm{C}$ for $30 \mathrm{~min}$, then they were filtered and washed with distilled water until neutral $\mathrm{pH}$. The functionalized MWCNTs (f-MWCNTs) were finally dried at $120^{\circ} \mathrm{C}$ and stored in a sealed container under vacuum prior to use. This procedure introduces oxygencontaining groups, in particular hydroxyl, carbonyl and carboxylic functionalities on the MWCNT surfaces as evidenced by XPS (Supporting information). The O/C ratios estimated for MWCNTs and f-MWCNTs are 0.026 and 0.218 , respectively.

FGS were produced from the adiabatical expansion[22] of graphite oxide at $1000^{\circ} \mathrm{C}$ under an inert atmosphere. Graphite oxide was synthesized from natural graphite according to the Brödie method.[23] FGS present hydroxyl, carbonyl and epoxy functional groups as observed by XPS (Supporting information) with an O/C ratio of 0.101. Full characterization of the FGS used in this study can be found elsewhere.[22] 


\subsection{Preparation of PU nanocomposite foams}

All foams were prepared from 100 phpp (parts per hundred parts of polyol) of Voranol 6150, 4 phpp of Voranol CP1421, 0.8 phpp of DEOA, 0.25 phpp of TEDA-L33B, 0.1 phpp of NIAX E-A-1, 0.05 phpp of FASCAT 4202, and 0.4 phpp of silicone SH 209 with the appropriate amount of water and isocyanate. Two series of PU foams were synthesized with $25 \%$ and $32.5 \%$ of HS and maintaining an isocyanate index of 100 . The formulations are detailed in Table 1.

Table 1. Flexible Polyurethane Foam Formulations.

\begin{tabular}{|l|c|c|}
\hline Sample & Voranate 2940 (phpp) & $\mathbf{H}_{2} \mathrm{O}$ (phpp) \\
\hline PU-25\% HS & 43.4 & 2.0 \\
\hline PU-32.5\% HS & 55.3 & 3.0 \\
\hline
\end{tabular}

For comparison purposes, we used a fixed amount of 0.5 phpp of carbon nanofillers (MWCNTs, f-MWCNTs and FGS), which corresponds to a total concentration in the final PU foam of $0.3 \mathrm{wt} .-\%$. Carbon nanofillers were added into the polyol (Voranol 6150), stirred for 6 hours at $2400 \mathrm{rpm}$ and ultra-sonicated for 20 minutes with a probe, within a water/ice bath to avoid temperature rising. Afterwards, the catalysts, surfactant and water were added to the polyol-carbon nanofiller dispersion and stirred again for 3 $\min$ at $2400 \mathrm{rpm}$. To reduce the reaction rate, both polyol mixture and isocyanate were maintained at $5^{\circ} \mathrm{C}$ until use, when the isocyanate was added to the mixture and stirred for 20 seconds.

\subsection{Characterization}

The infrared data were recorded on a Perkin-Elmer Spectrum One FT-IR spectrometer using the attenuated total reflectance (ATR) technique. Data were collected at $8 \mathrm{~cm}^{-1}$ resolution co-adding 6 scans per spectrum. The scanning time was about 2 seconds per spectrum and the reaction was followed for $1800 \mathrm{sec}$. An air background was performed at $4 \mathrm{~cm}^{-1}$ resolution and co-adding 256 scans prior to the execution of the reaction. The reactive mixture was placed in direct contact with the diamond crystal of the FT-IR 
where the foaming process occurred while monitoring the polymerization kinetics. Synchrotron experiments were carried out at the Spanish operated BM16-CRG beamline of the European Synchrotron Radiation Facility (ESRF) in Grenoble. The Xray beam energy was fixed to the Se-K to the edge $(\lambda=0.9795 \AA)$. Data were collected using a MarCCD165 detector (MarResearch, USA) in 1024x1024 pixels resolution mode (effective pixel size of $156 \mu \mathrm{m}$ ). The distance of the sample to the detector was set to $3420 \mathrm{~mm}$. The experimental procedure performed was as follows. The isocyanate was added to the polyol dispersions and mixed for $20 \mathrm{sec}$. Then, the reactive mixture was injected into the SAXS cell by means of a syringe. Samples were loaded in BM16CRG designed flat cells for which $25 \mu \mathrm{m}$ thick mica foils were used as windows. Experiment temperature $\left(\mathrm{T}=5^{\circ} \mathrm{C}\right)$ was achieved by circulating a fluid in the cell. 2-D to $\mathrm{I}(\mathrm{q})$ vs $\mathrm{q}$ data processing was performed using de Fit2D software and BM16-CRG routines. The reactions were recorded for 1500 sec. A clipped Gaussian random field model was used to obtain a visual representation of the developed matrix using the SAXS data. The raw data and the volume fraction, calculated from the invariant using absolutely calibrated data, were introduced into SAXSMorph program to generate a representative morphology, which was subsequently rendered using POVRay Software. Atomic force microscopy images were obtained using a tapping mode AFM (multimode Nanoscope Iva, Digital Instrument/Veeco) under ambient conditions. The oscillation frequency for the tapping mode was set to approximately $320 \mathrm{kHz}$. PU nanocomposite foams were compressed into solid elastomeric sheets at $140{ }^{\circ} \mathrm{C}$ and 200 bar in an electrically heated hydraulic press for $1 \mathrm{~h}$. The final thicknesses of the samples are $\sim 150$ $\mu \mathrm{m}$.

\section{Results and discussion}

The evolution of flexible PU foams involves many simultaneous reactions, though it is often assumed that there are only two main reactions, those of the isocyanate with the polyol and water, with no side reactions.[24, 25] The extent of the reaction in polyurethane foams is usually determined following the isocyanate conversion by FTIR.[26] The isocyanate absorption band appears at approximately $2270 \mathrm{~cm}^{-1}$ and it is normalized respect to an internal reference band that remains constant during the reaction $\left(\mathrm{CH}\right.$ stretch at $\left.2970 \mathrm{~cm}^{-1}\right)$, to compensate for the density change. Hence, the 
conversion is defined by

$$
p=1-\frac{A_{N C O}}{A_{0}}
$$

where $A_{N C O}$ is the integrated intensity of the normalized isocyanate absorbance band, and $A_{0}$ is the normalized isocyanate absorbance band at zero reaction time. The isocyanate conversion for PU-25\% HS and PU-32.5\% HS nanocomposite foams is shown in Figure 1.a and 1.b, respectively.

a

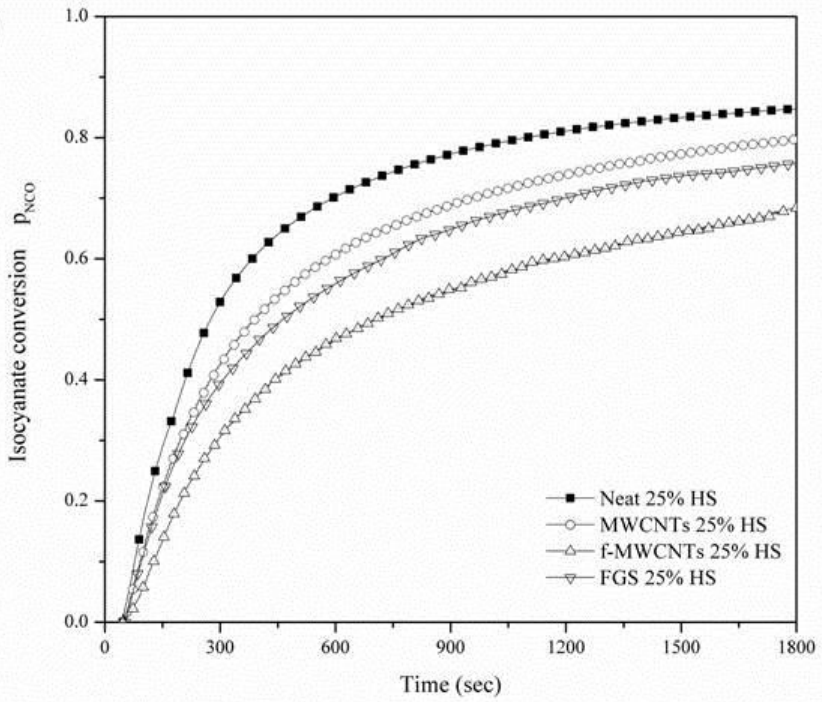

b

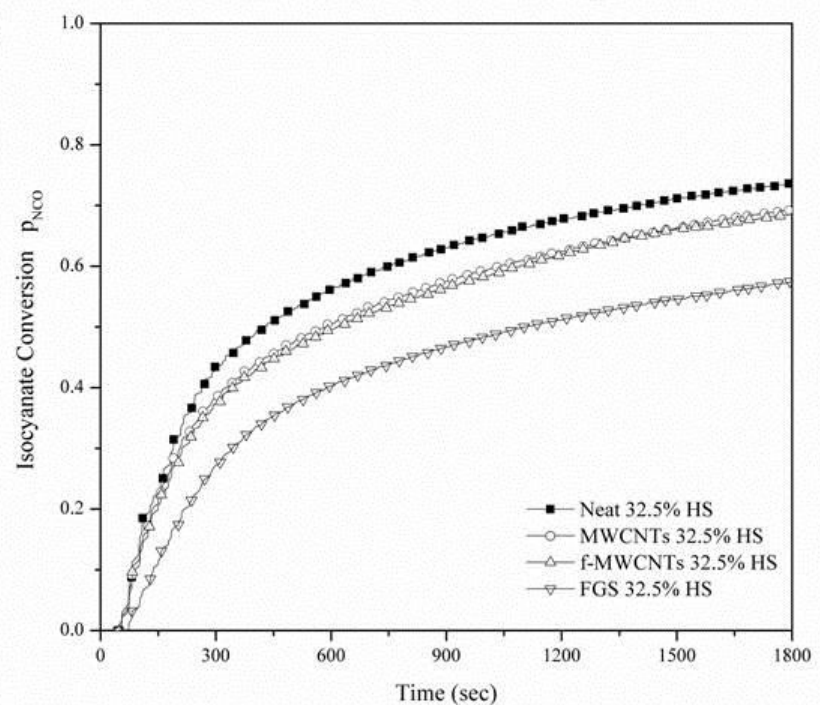

Fig. 1. Isocyanate conversion, $\mathrm{p}_{\mathrm{NCO}}$, as a function of time for a) PU-25\%HS and b) PU$32.5 \% \mathrm{HS}$ nanocomposite foams. 
The morphology and properties of polyurethane foams are strongly determined by the chemical reactions taking place during the curing reaction of the polymer. The kinetic data were determined taking into account the approximation of Li et al.[24] to a $n^{\text {th }}$ order kinetic approach, widely used for the modelling of polyurethanes.[25, 27, 28]

$n=1 \quad \ln (1-p)=-k_{0} t+C$

$n=2 \quad \frac{1}{(1-p)}=k_{0} t+C$

$n=3 \quad \frac{1}{(1-p)^{2}}=-k_{0} t+c$

where $p$ is the isocyanate conversion, $k_{0}$ is the kinetic constant, $t$ is the time value and $C$ is the integration constant. Equations (2-4) were applied to the isocyanate conversion data in order to find the appropriate kinetic model. In this study, the reaction of both sets of PU nanocomposite foams followed a third-order kinetics resulting from the autocatalytic effects of hydrogen bonding between the hydroxyl groups and the formed urethane and urea groups.[14, 29, 30]. Figure 2.a and 2.b illustrate the third order kinetic model applied for PU nanocomposite foams while the fitting results are summarized in Table 2. 

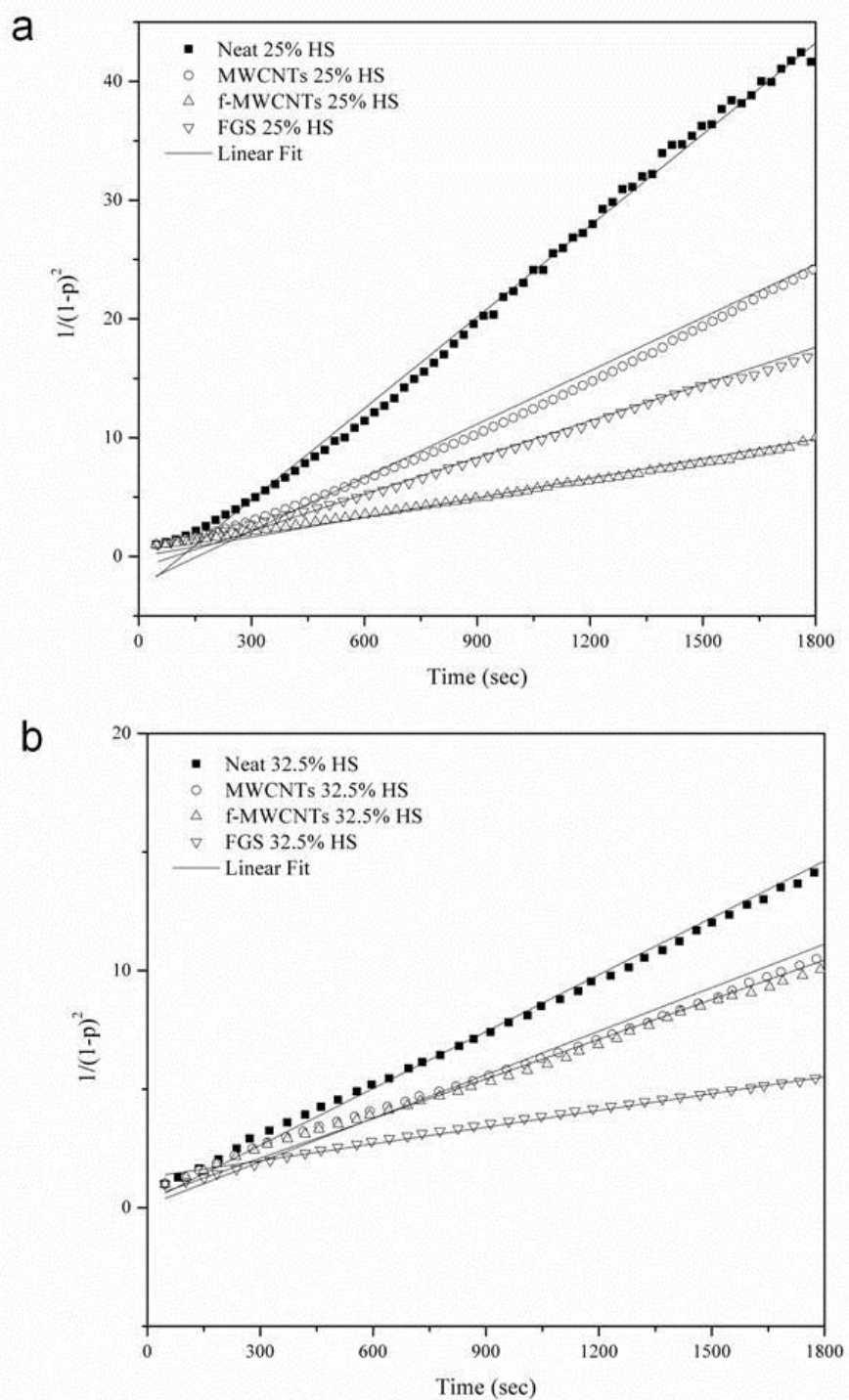

Fig. 2. Third-order kinetic model for a) PU-25\%HS and b) PU-32.5\%HS nanocomposite foams.

Table 2. Rate constants $k(\mathrm{sec})$ and fitting results $\left(r^{2}\right)$ for PU nanocomposite foams.

\begin{tabular}{|c|c|c|c|c|c|c|c|}
\hline & Sample & $k$ & $r^{2}$ & $k$ & $r^{2}$ & $k$ & $r^{2}$ \\
\hline \multirow{4}{*}{$\begin{array}{l}0 \\
0 \\
0 \\
10 \\
N\end{array}$} & Neat & $4.1 \cdot 10^{-4}$ & 0.779 & $2.0 \cdot 10^{-3}$ & 0.935 & 0.026 & 0.995 \\
\hline & MWCNTs & $4.2 \cdot 10^{-4}$ & 0.863 & $1.0 \cdot 10^{-3}$ & 0.976 & 0.015 & 0.998 \\
\hline & f-MWCNTs & $3.5 \cdot 10^{-4}$ & 0.913 & $0.9 \cdot 10^{-3}$ & 0.933 & 0.006 & 0.992 \\
\hline & FGS & $3.9 \cdot 10^{-4}$ & 0.879 & $1.0 \cdot 10^{-3}$ & 0.930 & 0.010 & 0.998 \\
\hline
\end{tabular}




\begin{tabular}{|c|c|c|c|c|c|c|c|}
\hline \multirow{4}{*}{ 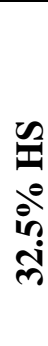 } & Neat & $3.5 \cdot 10^{-4}$ & 0.884 & $1.0 \cdot 10^{-3}$ & 0.970 & 0.008 & 0.999 \\
\hline & MWCNTs & $3.4 \cdot 10^{-4}$ & 0.918 & $1.0 \cdot 10^{-3}$ & 0.960 & 0.007 & 0.995 \\
\hline & f-MWCNTs & $3.3 \cdot 10^{-4}$ & 0.912 & $0.9 \cdot 10^{-3}$ & 0.936 & 0.006 & 0.998 \\
\hline & FGS & $2.5 \cdot 10^{-4}$ & 0.904 & $0.5 \cdot 10^{-3}$ & 0.973 & 0.002 & 0.998 \\
\hline
\end{tabular}

The evolution of the phase-separated morphology, characteristic of polyurethanes, implies the competition between the kinetics of polymerization, kinetics of phase separation and vitrification of hard segments.[11] Thus, the rate of reaction depends on the competitive reactions during the process, including the reactions with the functional groups present on the surface of the nanoparticles. Once these reactions have taken place, the mobility of the recently formed urea HS groups, depends on the local constraints. The reaction kinetics was strongly influenced by the HS content (Figure 1). The PU-25\% HS samples reached a maximum isocyanate conversion of about $80 \%$ at $1800 \mathrm{sec}$, while the isocyanate conversion only reached $70 \%$ or even lower for the PU$32.5 \%$ HS samples. This decrease in the rate and isocyanate conversion was ascribed to an increase in the viscosity of the system and, hence, a restriction of the chain mobility due to the larger HS content. This result agrees well with previously reported studies of the effect of HS content on the isocyanate conversion.[3, 31, 32]

The inclusion of carbon nanofillers also affected the rate of polymerization as can be observed in Figure 1. The mobility for carbon nanofiller systems was further hindered by both the viscosity effect of these nanoparticles and the obstruction on the formation and aggregation of new hydrogen bonds between the HS. In addition, the influence of the carbon nanoparticles on the rate of reaction varied for the different sets of PU foams but, in all cases, there was a decrease on the extent of reaction, as can be observed in Figure 1 and Table 2. In order to describe the effect of the carbon nanofillers we have to take into account two factors: the morphology of the nanofillers and the type and proportion of oxygen-bearing groups on their surface. Since MWCNTs and f-MWCNTs are rod-like nanoparticles while FGS are platelet-like nanofillers, the effect of the different specific surface area in the polymerization reaction of the PU foams could differ. Further studies are now underway to study this effect by analyzing different carbon-based nanofillers bearing the same functional groups. Here, we concentrated on 
the effect of the functional groups: $\mathrm{C}-\mathrm{OH}$ and $\mathrm{COOH}$ on the sidewalls of f-MWCNTs and $\mathrm{C}-\mathrm{OH}, \mathrm{COOH}$ and $\mathrm{C}-\mathrm{O}$ groups on FGS surface as determined by XPS (Supporting information).

Foams filled with raw MWCNTs lower the rate of the isocyanate conversion on both sets of PU foams. This slow down on the kinetics is attributed to the restriction of the mobility of the systems due to the presence of rigid nanoparticles, previously observed as the formation of a percolated rheology network. $[18,19]$ However, the deceleration is less pronounced for MWCNTs-32.5\% HS because the increase on HS content already significantly delayed the reaction. Besides the viscosity effect observed for pristine MWCNTs, the COOH groups on the surface of f-MWCNTs could also deactivate the tin catalyst of the isocyanate-polyol reaction.[33] Thus, slowing even further the kinetics of polymerization. Meanwhile, the sample filled with FGS presented an even slower polymerization reaction than f-MWNT and MWNT samples for high contents of HS. Previous studies demonstrated [34] that adsorbed polar molecules, such as water, on the graphene surface move towards the oxidized edges creating hydrogen bonds with the oxygen of the $\mathrm{C}-\mathrm{O}$ bonds $(\mathrm{H} \cdots \mathrm{O}-\mathrm{C})$. A charge transfer between graphene and the hydrogen atoms in water molecules is then created. The efficiency of this charge transfer increases with the number of water molecules. Hence, the drastic deceleration observed on PU-32.5\% HS filled with FGS could be attributed to the formation of hydrogen bonds with the $\mathrm{C}-\mathrm{O}$ groups on the surface of the graphene due to the additional water content of these foams. Consequently, the water molecules available for the isocyanate reaction are reduced and the reaction is slower compared to PU-25\% HS foams with the same content of FGS.

Synchrotron SAXS measurements were then used to analyze the influence of the hard segment content and the presence of the carbon nanoparticles on the formation of the microphase separated structure. Figure 3 shows a representative plot of scattered intensity, $I(q, t)$, against scattering vector, $q$, at different reaction times for PU-25\% HS foam. It can be observed, in all cases, that at the early stages of the reaction there was a homogeneous liquid present. Then, once the microphase separation transition was achieved, a scattering maximum can be detected and its intensity progressively increased until constant value was reached. This constant value can be related to the Berghmans point of the PU foams where vitrification freezes the morphology.[2] 


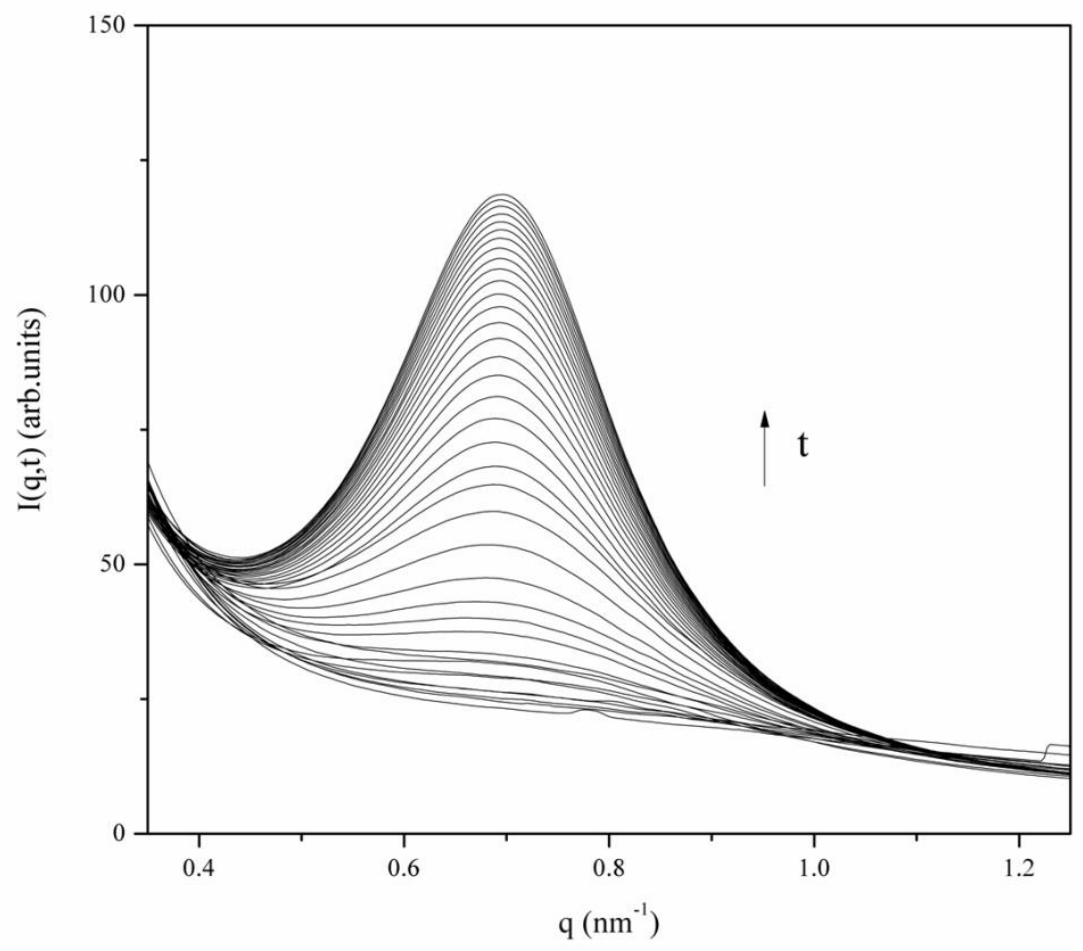

Fig. 3. Evolution of the scattered intensity, $I(q, t)$, vs the scattering vector, $q$, during the reaction time of PU-25\% HS foam.

Therefore, the maximum observed in $I(q)$ is indicative of the presence of a periodic structure within the systems. Equation 5, derived from Bragg's law, was applied in order to determine the average interdomain spacing $(d)$, which is inversely proportional to the scattering vector $q_{\max }$ calculated from the Lorentz corrected plot $I(q) q^{2}$ vs $q$.

$d=\frac{2 \pi}{q_{\max }}$

The evolution of the interdomain spacing was calculated from the first reliable $q_{\max }$ obtained from the $I(q) q^{2}$ vs $q$ plots to the end of the measurements $(1500 \mathrm{sec})$. Figures 4.a and 4.b show the development of $d$ as a function of time for PU-25\%HS and PU$32.5 \%$ HS nanocomposite foams, respectively. It was observed that the interdomain spacing continuously increased until the microphase separation was arrested by vitrification. This increase in the interdomain spacing could be related to the increase of the size of the urea hard segments.[11] It can be noticed that the trend followed by the rate of formation of the interdomain spacing is similar to the rate of isocyanate 
conversion analyzed by FT-IR for both sets of PU nanocomposite foams. Hence, there appears to be a correlation between the kinetics of polymerization and the kinetics of phase separation of hard segments. The values of the interdomain spacing $(d)$ are resumed in Table 3. However, it should be noted that for PU-32.5\% HS the development of the HS had not reached the plateau at the end of the measurement (Figure 4) and hence it would continue afterwards.
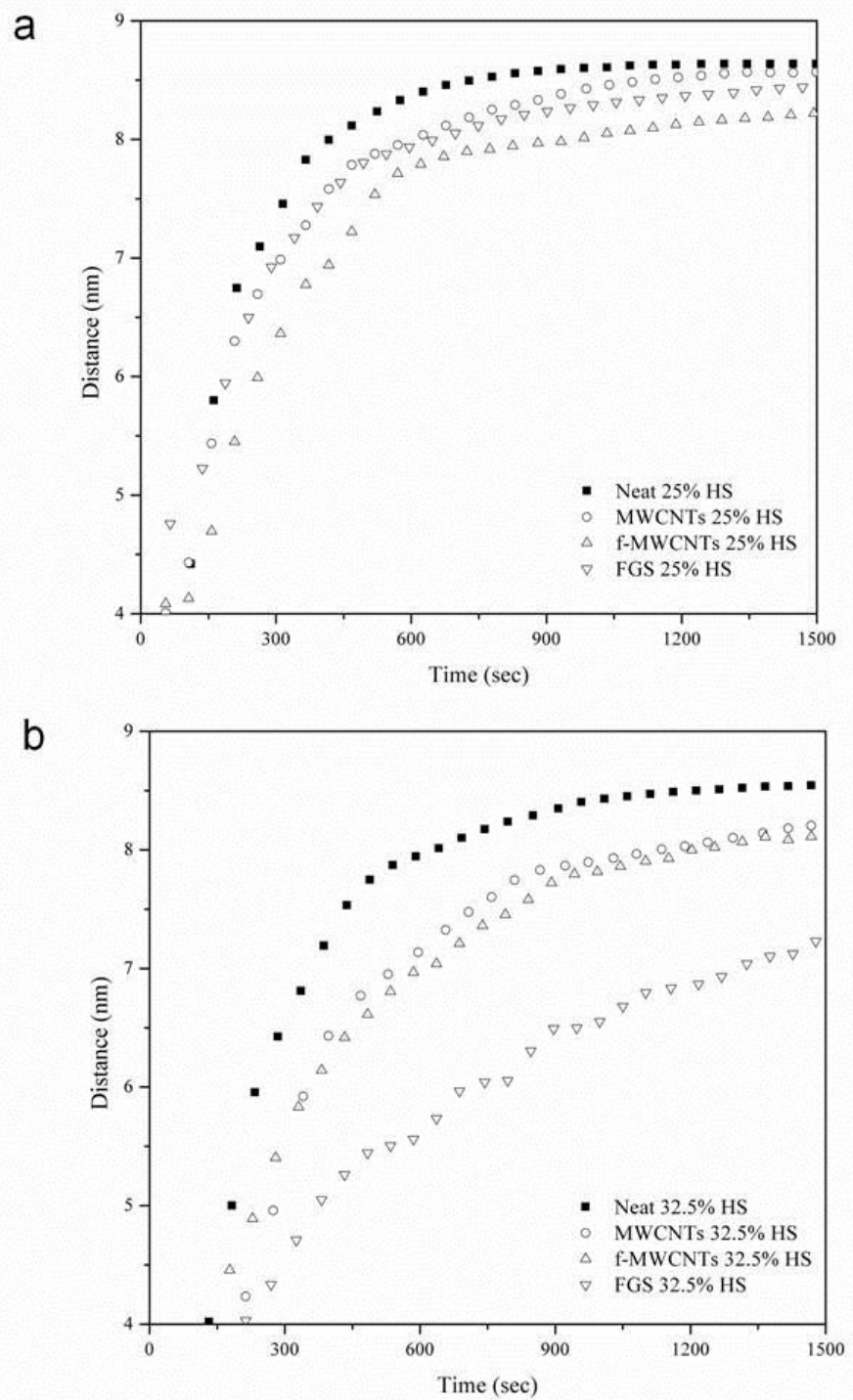

Fig. 4. Evolution of the interdomain spacing, $d$, with time for a) PU-25\% HS and b) PU$32.5 \%$ HS nanocomposite foams. 
Table 3. Interdomain spacing $(d)$ calculated from the Lorentz corrected plot and long period $(L p)$ estimated by the correlation function at $1500 \mathrm{sec}$.

\begin{tabular}{|l|l|c|c|}
\hline \% HS & Sample & $\boldsymbol{d}(\mathbf{n m})$ & $\boldsymbol{L p}(\mathbf{n m})$ \\
\hline $\mathbf{2 5}$ & Neat & 8.6 & 8.3 \\
\hline & MWCNTs & 8.5 & 8.0 \\
\hline & f-MWCNTs & 8.2 & 7.7 \\
\hline & FGS & 8.4 & 7.9 \\
\hline $\mathbf{3 2 . 5}$ & Neat & 8.5 & 8.1 \\
\hline & MWCNTs & 8.2 & 7.7 \\
\hline & f-MWCNTs & 8.1 & 7.6 \\
\hline & & 7.1 & 7.5 \\
\hline
\end{tabular}

The interdomain spacing could also be estimated using the one-dimensional correlation function $\chi(r)$ which is related to the electron density fluctuation within the system and is defined by Equation 6:

$\gamma(r)=\frac{1}{Q} \int_{0}^{\infty} I(q) q^{2} \cos (q r) d q$

In Figure 5, the correlation functions for the two PU nanocomposite foam systems at the end of the measurements are plotted. The one-dimensional correlation function showed periodicity for both PU foam series indicating a two-phase structure in the systems[6, 35-37] and the first maximum observed in the correlation curve is associated with the interdomain spacing or long period $\left(L_{p}\right)$. The values of the long period at $1500 \mathrm{sec}$ of reaction are reported in Table 3 and are in good agreement with those estimated using Bragg's law. The increase of the HS content and carbon nanotubes (MWCNTs and fMWCNTs) has little influence on the interdomain spacing.[6] However, the inclusion of 
FGS decreased this value for high contents of HS. As has been explained in the FT-IR results, the higher water content on these formulations enhanced the efficiency of the charge transfer between the graphene and the hydrogen atoms of the water molecules which would thus affect the phase-separated structure of the HS.
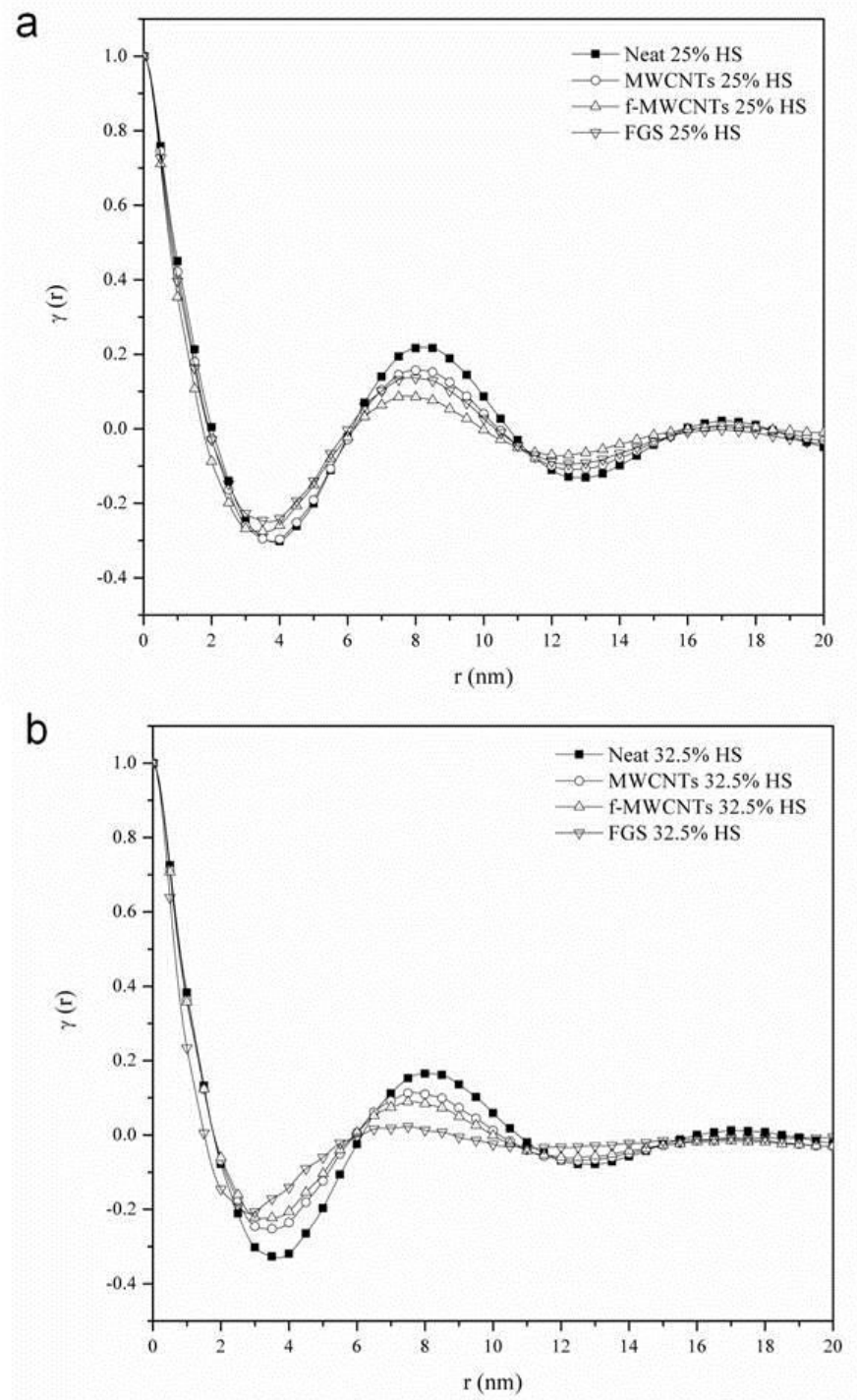

Fig. 5. One-dimensional correlation function $\chi(r)$ of the SAXS data for a) PU-25\% HS nanocomposite foams and b) PU-32.5\% HS nanocomposite foams.

The SAXS data were finally analyzed with a clipped random wave $(C R W)$ technique that provides a visual representation of the structure of the PU nanocomposite foams. This model has been widely used to analyze the morphology of other disordered bicontinuous phase materials, such as gels,[38] polymer blends,[39] aerogels,[40] and ionomers.[41] This model-independent approach provides representative 3D structures 
corresponding to the experimental scattering data.[42] Figure 6 shows the evolution of the morphologies (HS are represented as solid) at $250 \mathrm{sec}$ and $1500 \mathrm{sec}$ for PU-25\% HS and PU-32.5\% HS nanocomposite foams, respectively. (Detailed explanation of the analysis approach is provided in the Supporting Information). The deceleration observed for PU-32.5\% HS foams in the formation of the interdomain spacing respect to the PU-25\% HS foams is clearly observed on the $C R W$ results. At the early stages of the reaction $(250 \mathrm{sec})$, the HS domains formed for PU-32.5\% HS with carbon nanotubes and especially for FGS foams are lower compared to the PU-25\% HS foam. However, at $1500 \mathrm{sec}$ the Neat sample of PU-32.5\% HS is more interconnected than the Neat PU$25 \%$ HS, while foams with carbon nanofillers still present low concentration of HS domains and less interconnection (Table SI.2) which confirms the results obtained by other techniques.
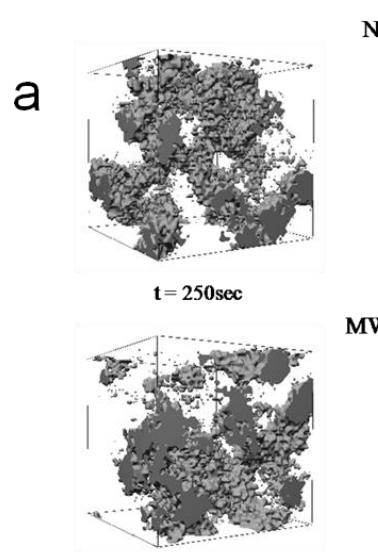

$\mathbf{t}=250 \mathrm{sec}$

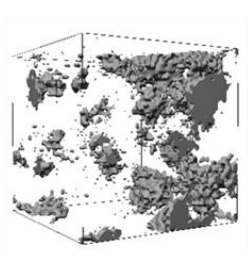

$\mathbf{t}=250 \mathrm{sec}$

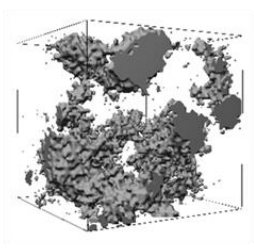

$\mathbf{t}=250 \mathrm{sec}$
MWCNT

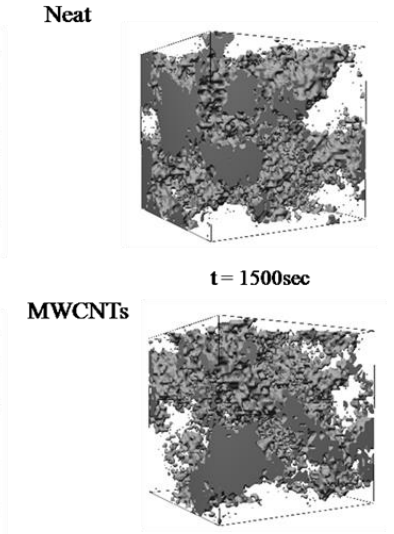

$t=1500 \mathrm{sec}$

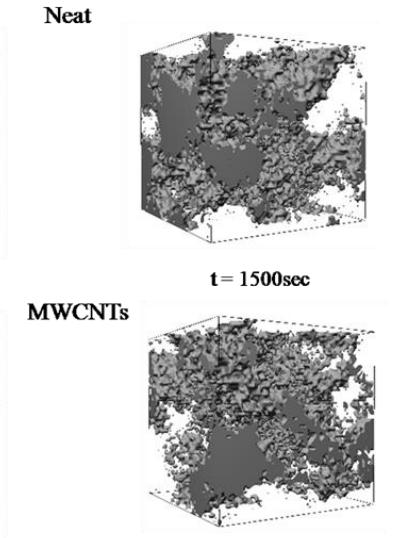

$\mathbf{t}=1500 \mathrm{sec}$

f-MWCNTs

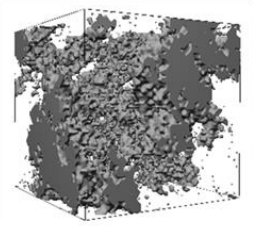

$t=1500 \mathrm{sec}$

FGS

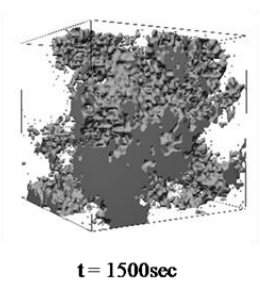

b

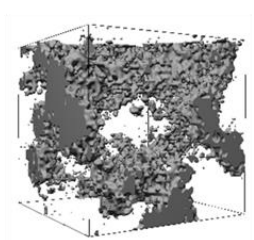

$t=250 \mathrm{sec}$

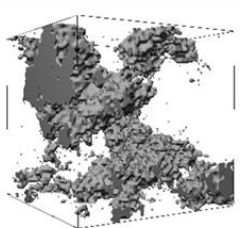

$\mathbf{t}=250 \mathrm{sec}$

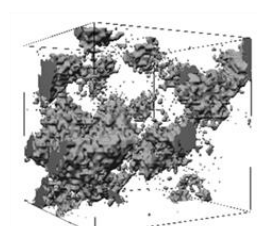

$\mathbf{t}=\mathbf{2 5 0 \mathrm { sec }}$

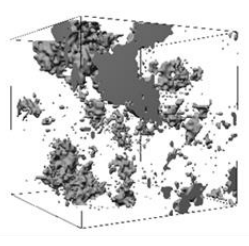

$t=250 \mathrm{sec}$
Neat

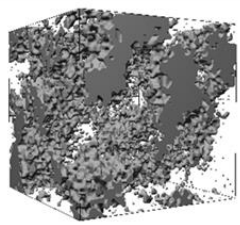

$t=1500 \mathrm{sec}$

MWCNTs

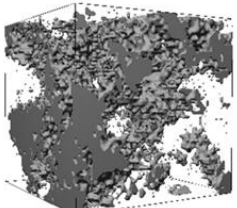

$\mathbf{t}=1500 \mathrm{sec}$

f-MWCNTs

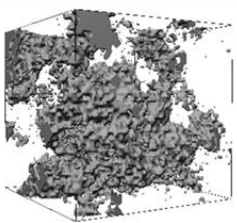

$t=1500 \mathrm{sec}$

FGS

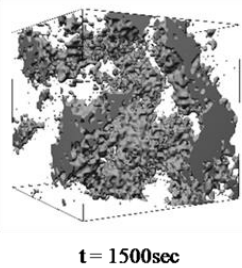

Fig. 6. 3D structure of the evolution of the microdomains at $250 \mathrm{sec}$ and $1500 \mathrm{sec}$ for a) PU-25\% HS and b) $32.5 \%$ HS nanocomposite foams. Cubes are $200 \AA$ per side. 
Atomic force microscopy (AFM) was used to qualitatively examine the presence of two-phases in the morphology of FPU nanocomposite foams. In the phase images (Figure 7), two different coloured areas are observed: light ones for hard segment domains and dark areas for soft segment domains.[43-45] The overall phase scale of the images was adjusted to $25^{\circ}$ in order to clarify the foam morphology and facilitate the comparison between samples.

As the hard segment content increases, AFM images reveal an increase on the hard domain connectivity and the formation of large structures.[44, 46, 47] It has previously been reported $[42,46]$ that larger structures are aggregates of smaller hard domains. The AFM images showed an estimated interdomain spacing of 10-12 nm. Since SAXS gives values of the interdomain distances of only periodic structures, [43, 48] the results of both techniques are in good agreement. Nevertheless, it appears that the formation of the urea aggregates on nanocomposite foams is influenced by the presence of CNPs. Song et al. [49] suggested that formation of aggregates is related to the reaction kinetics and the presence of fillers in the matrix. Therefore, fast reaction rates form aggregates with smaller average size due to the rapid aggregation behavior, while the presence of fillers inhibits the aggregation of hard domains which is observed in foams filled with CNPs. On the other hand, it is observed on the AFM images that foams with MWCNTs display a denser urea aggregates and clearer phase contrast compared to foams filled with functionalized CNPs, which indicates a better microphase separation.[50, 51] This observation suggests that the functional groups on the surface of the carbon nanofillers are creating interactions with the hard domains. 

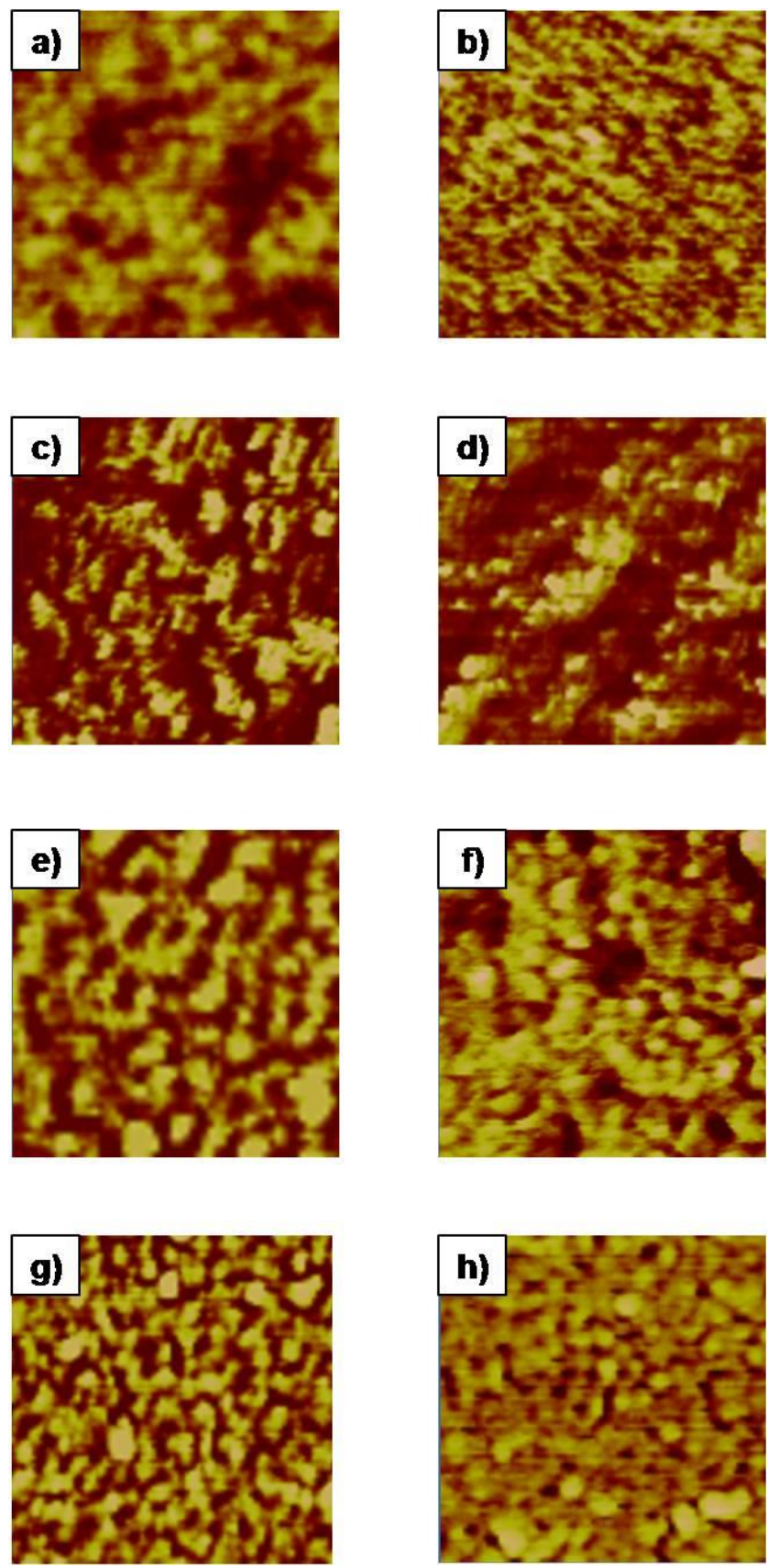

Fig. 7. Tapping mode AFM phase images. Images are displayed at a total phase scale of $25^{\circ}$ and the scan width is $500 \mathrm{~nm}$. Images a), c), e) and g) are Neat, MWCNTs, fMWCNTs and FGS FPU-25\% HS nanocomposite foams, respectively. Images b), d), f) and h) are Neat, MWCNTs, f-MWCNTs and FGS FPU-32.5\% HS nanocomposite foams, respectively. 


\section{Conclusions}

The polymerization reaction and the development of the phase-separated structure of flexible PU foams are strongly influenced both by HS content and by the presence of carbon nanoparticles with different morphologies and surface bearing groups. FT-IR analysis confirmed a deceleration on the kinetics of polymerization due to the increase of the hard segment content. Meanwhile, synchrotron SAXS analysis confirmed the delay of the morphology development being the rate of evolution of the interdomain spacing slower for PU-32.5\% HS nanocomposite foams. Thus, the kinetics of polymerization and the kinetics of phase separation of hard segments follow a parallel trend. The effect of the nanoparticles on the polymerization and microphase structure depended on their influence on the reactants viscosity, the type of functional groups on their surface and the water/isocyanate ratio. Current studies are underway to establish a possible effect of the nanofiller morphology. The visualizations of the CRW model images clarify the results obtained by FT-IR spectroscopy and SAXS. Meanwhile, AFM images showed the influence of the nanoparticles on the two-phase morphology of PU nanocomposite foams.

\section{Acknowledgements}

The authors gratefully acknowledge the financial support of the Spanish Ministry of Science and Innovation (MICINN) through MAT 2010-18749 and the $7^{\text {th }}$ Framework Program of E.U. through HARCANA (NMP3-LA-2008-213277). MMB and MMG also acknowledge the FPI and JAE-Pre programs from MICINN and CSIC, respectively. The authors thank Dr. Angel Marcos-Fernández for helpful discussions and Dr. Gema Rodriguez for AFM images.

\section{Electronic Supplementary Information (ESI) available:}

Figure SI-1. High resolution $\mathrm{C}_{1 \text { s }}$ and $\mathrm{O}_{1 \mathrm{~s}}$ XPS spectra of a) MWCNTs, b) f-MWCNTs and c) FGS. Curves inside are deconvoluted curve fits of the spectra. Figure SI-2: Representative $2 D$ image generated by SAXSMorph at $250 \mathrm{sec}$ and $1500 \mathrm{sec}$. Box size is $200 \AA$ and the volume fraction $25 \%$. Grey color represents the HS. Table SI-1. Assignment of binding energy (BE) and atomic bond contribution after the deconvolution of the XPS spectra of MWCNTs, f-MWCNTs and FGS. Table SI-2: Average size and connectivity of the structures from image analysis of the $C R W$ morphologies. 


\section{References}

1. Cao X, Lee LJ, Widya T, and Macosko C. Polymer 2005;46(3):775-783.

2. Klempner D and Sendijarevic V. Handbook of polymeric foams and foam technology: Hanser Publishers, 2004.

3. Artavia LD and Macosko CW. J. Cell. Plastics.1990;26(6):490-511.

4. Elwell MJ, Mortimer S, and Ryan AJ. Macromolecules 1994;27(19):5428-5439.

5. Neff RA and Macosko CW. Rheologica Acta 1996;35:656-666.

6. Li Y, Ren Z, Zhao M, Yang H, and Chu B. Macromolecules 1993;26(4):612622.

7. Li Y, Kang W, Stoffer JO, and Chu B. Macromolecules 1994;27(2):612-614.

8. Koberstein JT and Russell TP. Macromolecules 1986;19(3):714-720.

9. Miller JA, Lin SB, Hwang KKS, Wu KS, Gibson PE, and Cooper SL. Macromolecules 1985;18(1):32-44.

10. Pongkitwitoon S, Hernández R, Weksler J, Padsalgikar A, Choi T, and Runt J. Polymer 2009;50(26):6305-6311.

11. Li W, Ryan AJ, and Meier IK. Macromolecules 2002;35(13):5034-5042.

12. Bras W, Derbyshire GE, Bogg D, Cooke J, Elwell MJ, Komanschek BU, Naylor S, and Ryan AJ. Science 1995;267(5200):996-999.

13. Elwell MJ, Ryan AJ, Grünbauer HJM, and Van Lieshout HC. Macromolecules 1996;29(8):2960-2968.

14. Cunliffe AV, Davis A, Farey M, and Wright J. Polymer 1985;26(2):301-306.

15. Harikrishnan G, Patro TU, and Khakhar DV. Ind. Eng. Chem. Res. 2006;45(21):7126-7134.

16. Wilkinson AN, Fithriyah NH, Stanford JL, and Suckley D. Macromol. Symp. 2007;256(1):65-72.

17. Verdejo R., Stampfli R., Alvarez-Lainez M., Mourad S., Rodriguez-Perez M. A., Bruhwiler P. A., Shaffer M., Compos. Sci. Technol. 2009;69(10),1564-1569.

18. Bernal MM, Lopez-Manchado MA, and Verdejo R. Macromol. Chem. Phys 2011;212(9):971-979.

19. Bernal MM, PhD Thesis, 2012 Universidad Politecnica de Valencia Spain

20. Singh C, Shaffer MS, and Windle AH. Carbon 2003;41(2):359-368.

21. Verdejo R, Lamoriniere S, Cottam B, Bismarck A, and Shaffer M. Chem. Commun. 2007(5):513-515.

22. Verdejo R, Barroso-Bujans F, Rodriguez-Perez MA, de Saja JA, and LopezManchado MA. J. Mater. Chem. 2008;18(19):2221-2226.

23. Brödie BC. Philosophical Transactions of the Royal Society of London 1859;149:249-259. 
24. Li S, Vatanparast R, and Lemmetyinen H. Polymer 2000;41(15):5571-5576.

25. Chattopadhyay DK, Prasad PSR, Sreedhar B, and Raju K. Prog. Org. Coat. 2005;54(4):296-304.

26. Heintz AM, Duffy DJ, Nelson CM, Hua Y, Hsu SL, Suen W, and Paul CW. Macromolecules 2005;38(22):9192-9199.

27. Maji PK and Bhowmick AK. J. Polym. Sci., Part A: Polym. Chem. 2009;47(3):731-745.

28. de Lima V, Pelissoli ND, Dullius J, Ligabue R, and Einloft S. J. Appl. Polym. Sci. 2010;115(3):1797-1802.

29. Eceiza A, de la Caba K, Gascon V, Corcuera MA, and Mondragon I. Eur. Polym. J. 2001;37(8):1685-1693.

30. Han JL, Yu CH, Lin YH, and Hsieh KH. J. Appl. Polym. Sci. 2008;107(6):38913902.

31. Garcia-Moreno F, Fromme M, and Banhart J. Advanced Engineering Materials 2004;6(6):416-420.

32. Ryan AJ. Polymer 1990;31(4):707-712.

33. Draye AC and Tondeur JJ. J. Mol. Catal. A: Chem. 1999;138(2-3):135-144.

34. Berashevich J and Chakraborty T. Physical Review B 2010;81(20).

35. Chang SL, Yu TL, Huang CC, Chen WC, Linliu K, and Lin TL. Polymer 1998;39(15):3479-3489.

36. Strobl GR and Schneider M. J. Polym. Sci., Part B: Polym. Phys. 1980;18(6):1343-1359.

37. Roe R-J. Methods of X-ray and Neutron Scattering in Polymer Science: Oxford University Press: New York, 2000.

38. Gommes CJ and Roberts AP. Phys. Rev. E 2008;77(4).

39. Jinnai H, Hashimoto T, Lee D, and Chen SH. Macromolecules 1997;30(1):130136.

40. Quintanilla J, Reidy RF, Gorman BP, and Mueller DW. J. Appl. Phys. 2003;93(8):4584-4589.

41. Aieta NV, Stanis RJ, Horan JL, Yandrasits MA, Cookson DJ, Ingham B, Toney MF, Hamrock SJ, and Herring AM. Macromolecules 2009;42(15):5774-5780.

42. Ingham B, Li H, Allen EL, and Toney MF. J. Appl. Crystallogr. 2011;44(1):221224.

43. McLean RS and Sauer BB. Macromolecules 1997;30(26):8314-8317.

44. Garrett JT, Runt J, and Lin JS. Macromolecules 2000;33(17):6353-6359.

45. Zhang L, Jeon HK, Malsam J, Herrington R, and Macosko CW. Polymer 2007;48(22):6656-6667.

46. O'Sickey MJ, Lawrey BD, and Wilkes GL. J. Appl. Polym. Sci. 2002;84(2):229- 
243.

47. Aneja A and Wilkes GL. Polymer 2003;44(23):7221-7228.

48. Kaushiva BD and Wilkes GL. Polymer 2000;41(18):6987-6991.

49. Song M, Xia HS, Yao KJ, and Hourston DJ. Eur. Polym. J. 2005;41(2):259-266.

50. Zhang S, RenLiu, Jiang J, Yang C, Chen M, and Liu X. Prog. Org. Coat. 2011;70(1):1-8.

51. Xia H, Song M, Zhang Z, and Richardson M. J. Appl. Polym. Sci. 2007;103(5):2992-3002. 